\title{
ON THE POSSIBILITY OF LONG-TERM FORECASTING OF SEASONAL HYDROMETEOROLOGICAL PHENOMENA
}

\author{
Alimpieva M.A., Morozova S.V. \\ Saratov national research state University named after N.G. Chernishevski \\ Corresponding authors: swetwl@yandex.ru
}

Citation: M.A. Alimpieva, S.V. Morozova 2020. On the possibility of long-term forecasting of seasonal hydrometeorological phenomena // Environmental dynamics and global climate change. V. 11. N.2. P. 73-78.

DOI: $10.17816 /$ edgcc21202

Text of the article in English: https://edgccjournal.org/EDGCC/article/view/19010

In present paper the expanding application possibility of physical-statistical methods in long-term forecast are viewed. A nonparametric discriminate analyzing model has been constructed on the South-East of EPR (European part of Russia). The model is based on consideration of asynchronous bonds between the condition of circulating systems of the Atlantic-Eurasian hemisphere sector and the period of seasonal hydrotermeological events onset on South-East of EPR. This model allows distinguishing three cluster areas which associate with three phases of predicted event; commonly, only two cluster areas are distinguished. We would like to present results of predictions testing of training and control sets. The conclusion of our model region appliance effectiveness is also represented in paper.

Key words: long-term weather forecast, centers action of the atmosphere, physical-statistical model, discriminate analysis

Рассматриваются вопросы расширения применения физико-статистических методов в долгосрочном прогнозировании. На основании учета асинхронных связей между состоянием циркуляционных систем Атлантико-Евразийского сектора полушария и сроками наступления сезонных гидрометеорологических явлений на юго-востоке ЕЧР построена модель непараметрического дискриминантного анализа, позволяющая разделять не две, что делается традиционно, а три кластерные области, соответствующие трем фазам прогнозируемого явления. Приводятся результаты тестирования прогнозов на обучающей и контрольной выборках. Делается вывод об эффективности применения модели в региональном прогнозировании.

Ключевые слова: долгосрочный прогноз, центры действия атмосферы, физико-статистическая модель, дискриминантный анализ

\section{BBEQ탵}

Проблема прогноза погоды на долгие сроки является довольно сложной научной задачей. Несомненные успехи, достигнутые в области долгосрочного прогнозирования в начале нашего века, безусловно, связаны с внедрением в оперативную практику глобальных численных моделей (ПЛАВ, МОЦАТ63L25 и др.) такими ведущими отечественными и мировыми центрами как Гидрометцентр России, Северо-Евразийский климатический центр, Главная геофизическая обсерватория им. А.И. Воейкова, Европейский центр среднесрочного прогнозирования погоды. Однако оправдываемость долгосрочных прогнозов все еще остается довольно низкой. При этом модельная прогностическая продукция обычно представляется полями аномалий метеовеличин или их характеристик, осредненных на различных временных интервалах - от нескольких суток до месяца, и, как правило, в вероятностной форме.
При этом остается необходимость в прогнозировании не только общего фона метеовеличин или его аномалий, но и в прогнозирования различных гидрометеорологических явлений, например, засух, типов весен, дат перехода средней суточной температуры воздуха через определенные пределы, сроков установления и схода снежного покрова, наступления и прекращения дождливых или сухих периодов и т.п. В настоящее время решение подобных задач в рамках гидродинамического моделирования не представляется возможным [Угрюмов, 2006], поэтому для этих целей все активнее привлекаются синоптические и физико-статистические методы [Вильфанд с соавт., 2017; Крыжов, 2012; Садоков с соавт., 2012]. Отметим, что глобальные численные модели всегда нуждаются в региональной статистической коррекции [Вильфанд с соавт. 2007; Мирвис с соавт., 2017]. 


\section{3АЯАЧИ ИССПEЯOBAHИЯ}

В настоящей публикации поставлена задача исследовать возможность долгосрочного прогнозирования сезонных гидрометеорологических явлений на основе учета дальних асинхронных связей циркуляционных систем Атлантико-Евразийского сектора полушария с различными характеристиками гидрометеорологического режима на юго- востоке Европейской части России. В качестве объекта прогнозирования выбраны даты перехода средней суточной температуры воздуха через $0^{\circ} \mathrm{C}, 5^{\circ} \mathrm{C}$ и $10^{\circ} \mathrm{C}$. Поскольку в данном случае важно знать не столько строго числовое значение предиктанта, а сколько возможность превышения (непревышения) им определенного критерия или осуществление той или иной фазы (класса), то для построения прогностических зависимостей использовалась модель непараметрического дискриминантного анализа. Отметим, что до настоящего времени при прогнозировании различных элементов погоды с применением дискриминантной модели использовалось двухфазное разделение предиктанта [Адрианова, 1971; Байдал и Неушкин, 1978]. В настояшем же исследовании дискриминантный анализ применяется для разделения трех фаз явления.

Поскольку технология применения дискриминантной модели идентична для всех объектов прогнозирования, покажем ее применение на примере прогноза даты перехода средней суточной температуры воздуха через $0^{\circ} \mathrm{C}$.

Районом исследования стала Саратовская область, являющаяся важным аграрным регионом России. Территория отличается высокой степенью континентальности климата, следствием чего является большая изменчивость погодного режима. Дата перехода через $0^{\circ} \mathrm{C}$ особенно важна для аграриев, поскольку именно эта дата по большей части определяет условия снеготаяния и накопление продуктивной влаги в почве к началу весенних полевых работ, а также развитие растений в начальные, самые ответственные для формирования урожая, фазы вегетации.

\section{ИНФОРМАЧИОНHАЯ БАЗА ИCСПEООВАНИЯ}

В качестве информационной базы исследования использовались данные о срочных и средних суточных значениях температуры воздуха по восьми станциям Саратовской области, расположенным в долине Волги (Саратов Юго-Восток, Октябрьский городок, Маркс, Вольск, Хвалынск, Золотое, Александров Гай, Новоузенск), которые характеризуются самыми ранними датами перехода средней суточной температуры через $0^{\circ} \mathrm{C}$ согласно климатическому районированию
Саратовской области [Атлас..., 1978]. На всей остальной территории, за исключением северных правобережных районов, даты перехода в многолетнем среднем наступают на три дня позже. В северных районах Правобережья области устойчивый переход осуществляется позже на шесть дней относительно территории в долине Волги.

Расчетная схема построения прогноза привязана именно к самым ранним датам перехода. Относительно этого заметим, что определенным новшеством разработанной авторами прогностической схемы можно считать «нарушение» традиционного алгоритма долгосрочного прогнозирования. Обычно прогноз выступает уточнением климатического фона, и все прогностические характеристики (аномалии) рассчитываются относительно средней многолетней (климата). В нашем случае климат выполняет не базисную, а корректируюшую функцию, то есть климатические закономерности (в данном случае районирование) позволяют адаптировать прогностическое решение ко всем остальным районам области. Определение дат устойчивых переходов проводилось авторами по разработанной оригинальной программе «DATAPEREHOD», составленной в соответствии с рекомендациями [Руководство...,1984]. Данные о температуре брались за 48-летний временной интервал с 1971 по 2018 гг. с сайта ВНИИГМИ-МЦД [Данные..., 2017] и из оригинальных архивов лаборатории агрометеорологии научно-исследовательского института сельского хозяйства Юго-Востока (НИИСХ ЮВ).

Циркуляционными структурами, характеристики которых использовались в построении расчетной схемы стали центры действия атмосферы (ЦДА) Атлантико-Евразийского сектора полушария, причем именно те, состояние которых испытывает заметную пространственно-временную изменчивость [Морозова, 2011]. Это Исландский циклон (ИМ) и зимний Азиатский (Сибирский) антициклон (CM). В качестве исходных данных в модель вводились не сами характеристики ЦДА (широта, долгота, давление в центре), а аномалии, которые рассчитывались как алгебраические отклонения от средних многолетних значений. Данные о состоянии ШДА с 1971 по 2010 гг. были позаимствованы из электронного приложения к Справочной монографии [Неушкин с соавт., 2013]. Характеристики ШДА с 2011 по 2018 гг. снимались со средних карт, размещенных на $\mathrm{ftp}$-сервере Гидрометцентра.

Выявление прогностических зависимостей проводились с помощью прикладного пакета программ «STATISTICA». Применялась процедура последовательного включения предикторов, количество переменных определялось по статистическим оценкам набора параметров $\left(R^{2}\right.$, частная $\lambda, F, p$-level $\left._{\max }\right)$. 


\section{METOのИKA}

Решение задачи базируется на теоретических разработках о дальних (teleconnection) и асихронных связях циркуляционных систем с элементами погоды в различных географических районах. Во многих работах [Крыжов,2013; Садоков с соавт,, 2012; Randall et al.,1998; Wallace and Gutzler,1981] показано, что между различными погодными режимами и циркуляционными индексами имеют место довольно устойчивые «дальние» связи, проявляющиеся не только в синхронном, но и асинхронном плане. Однако введение в прогностические модели только индексов, учитывающих интенсивность циркуляционных структур, существенно обедняет и модели, и ограничивает прогностические возможности самих объектов циркуляции. Поэтому авторы, помимо интенсивности выбранных центров действия атмосферы, в физико-статистической модели использовали и изменение пространственной локализации их центров.

При разработке прогностических указаний проводилась серия независимых статистических экспериментов с целью определения оптимальной заблаговременности принятия прогностического решения. На основании полученных оценок и с учетом практических запросов оптимальным промежутком выбран двухмесячный интервал.

\section{OCHOBHЫIE PEЗУПЫTATЫ}

Статистика дат перехода через $0^{\circ} \mathrm{C}, 5^{\circ} \mathrm{C}$ и $10^{\circ} \mathrm{C}$ показала, что наибольшую временную изменчивость имеет дата перехода через $0^{\circ} \mathrm{C}$ $(\sigma=5.02, C v=0.03)$, а наименьшую - через $10^{\circ} \mathrm{C}$

Таблица 1. Статистические характеристики преАикторов

\begin{tabular}{|c|c|c|c|c|}
\hline Предиктор & $\boldsymbol{R}^{2}$ & F-статистика & р-уровень & Частная $\lambda$ \\
\hline$x_{1}$ & 0.694 & 12.230 & 0.0007 & 0.380 \\
\hline$x_{2}$ & 0.734 & 6.269 & 0.0105 & 0.545 \\
\hline$x_{3}$ & 0.739 & 7.121 & 0.0066 & 0.513 \\
\hline$x_{4}$ & 0.778 & 6.872 & 0.0076 & 0.522 \\
\hline
\end{tabular}

$F_{\mathrm{Kp}}=3.8726$

Таблица 2. Значения коэффициентов разАеляющих функций и характеристика преАиктора

\begin{tabular}{|c|c|c|c|}
\hline \multirow{2}{*}{ Предиктор } & \multicolumn{2}{|c|}{ Значения коэффициентов } & \multirow{2}{*}{ Физическая характеристика преликтора } \\
\cline { 2 - 3 } & $L_{1}$ & $L_{2}$ & \multirow{2}{*}{ аномалия давления ИМ в январе } \\
\hline$x_{1}$ & 0.21 & 0.19 & аномалия долготы СМ в октябрег \\
\hline$x_{2}$ & 0.01 & 0.16 & аномалия широты ИМ в ноябре \\
\hline$x_{3}$ & -0.31 & -0.01 & аномалия долготы ИМ в ноябре \\
\hline$x_{4}$ & -0.07 & 0.06 & - \\
\hline Константа & 0.33 & -0.35 & \\
\hline
\end{tabular}

IC - Icelandic cyclone,

SA - winter Siberian (Asian) anticyclone $(\sigma=2.94, C v=0.02)$. Вариативность даты устойчивого перехода средней суточной температуры через $5^{\circ} \mathrm{C}$ такова: $\sigma=4.81, C v=0.03$. Поскольку прогностические разработки представлены только для даты перехода через $0^{\circ} \mathrm{C}$, то укажем, что самое раннее значение этой даты наблюдалось в 2008 году, когда переход через этот предел осуществился 23 февраля, а самый поздний имел место в 1983 году, переход через $0^{\circ} \mathrm{C}$ произошел 16 апреля (по данным метеостанции Саратов Юго-Восток). Таким образом, разброс крайних значений состав-

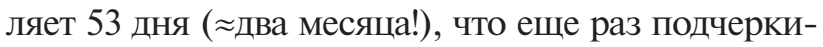
вает важность разработки методических прогнозов сезонных гидрометеорологических явлений.

В качестве потенциальных предикторов исследовались выбранные характеристики указанных центров действия предыдущего осенне-зимнего периода за исключением февраля. Внедрение в модель характеристик ЦДА в феврале делает заблаговременность прогноза практически нулевой, в результате чего прогноз становится не актуальным.

В результате расчетов выбраны четыре предиктора, параметры статистического оценивания которых приведены в таблице 1.

Как видно из таблицы, модель описывает около $75 \%$ изменчивости предиктанта, причем но устойчивы. По $F$-статистике везде имеем превышение его критического значения. По значению частной лямбда, показывающей вклад одиночного предиктора в разделение объектов, заключаем, что прогноз по группе факторов намного надежнее. В таблице 2 представлены физические характеристики предикторов и значения коэффициентов ЛДФ. От общего вида линейной дискриминантной функции (ЛДФ): значения коэффициента детерминации доволь- 


$$
L=b_{0}+b_{1} x_{1}+b_{2} x_{2}+\ldots+b_{n} x_{n}
$$

где $b_{0}-$ свободный член уравнения,

$b_{n}$ - коэффициенты линейной дискриминантной функции,

$x_{n}-$ (переменные) предикторы.

перейдем к стандартизованным уравнениям:

$L_{1}=0.33+0.21 x_{1}+0.01 x_{2}-0.31 x_{3}-0.07 x_{4}$

$L_{2}=-0.35+0.19 x_{1}+0.16 x_{2}-0.01 x_{3}+0.06 x_{4}$

Как видно из первого уравнения, наиболее значимыми для прогноза являются интенсивность Исландского минимума в январе и его смещение по меридиану в ноябре. По второму уравнению заметим, что наиболее сильно оказывают влияние на предиктант также интенсивность Исландского циклона в январе и смещение зимнего Азиатского антициклона вдоль круга широты в октябре.

Прогностические уравнения составлены на материале 1971 - 2010 гг. Значения ЛДФ для 2011 - 2018 гг. рассчитаны по полученным уравнениям по данным о состоянии центров действия атмосферы. На рисунке представлен график для прогноза характеристики даты весеннего перехода средней суточной температуры воздуха через $0^{\circ} \mathrm{C}$. На рисунке видно хорошее разделение фаз предиктанта.

Для определения качества предполагаемого метода необходимо оценить оправдываемость данного способа прогноза. Этот вид долгосрочного прогнозирования относится $\mathrm{K}$ категории многофазовых прогнозов. Методики оценивания многофазных $(>2)$ прогнозов, в отличие от двухфазных, разработаны недостаточно. Некоторые методические наработки можно найти в [Хандожко, 2005; Wilks, 1995]. В таблице 3 представлена матрица сопряженности, адаптированная к трехфазному прогнозу, в которую помещены результаты испытаний прогнозов на зависимом материале (числитель дроби) и с включением данных испытаний модели на независимой выборке (знаменатель дроби).

По этой таблице легко определить число случаев оправдавшихся прогнозов и дифференцированно рассмотреть неоправдавшиеся случаи - сколько прогнозов ранних переходов попало в область поздних, сколько поздних переходов попало в область нормальных и т.д. Например, из пяти неоправдавшихся прогнозов ранних дат перехода два попало в область нормальных и три - в область поздних.

Как приближенная мера качества прогнозов явлений погоды используется показатель $P$, показывающий долю оправдавшихся прогнозов к их общему числу. Доля оправдавшихся прогнозов составила $87.5 \%$ по зависимой выборке и $77 \%$ с учетом независимых испытаний. Значения показателя 9 оказались равными 0.78 и 0.54 соответственно.

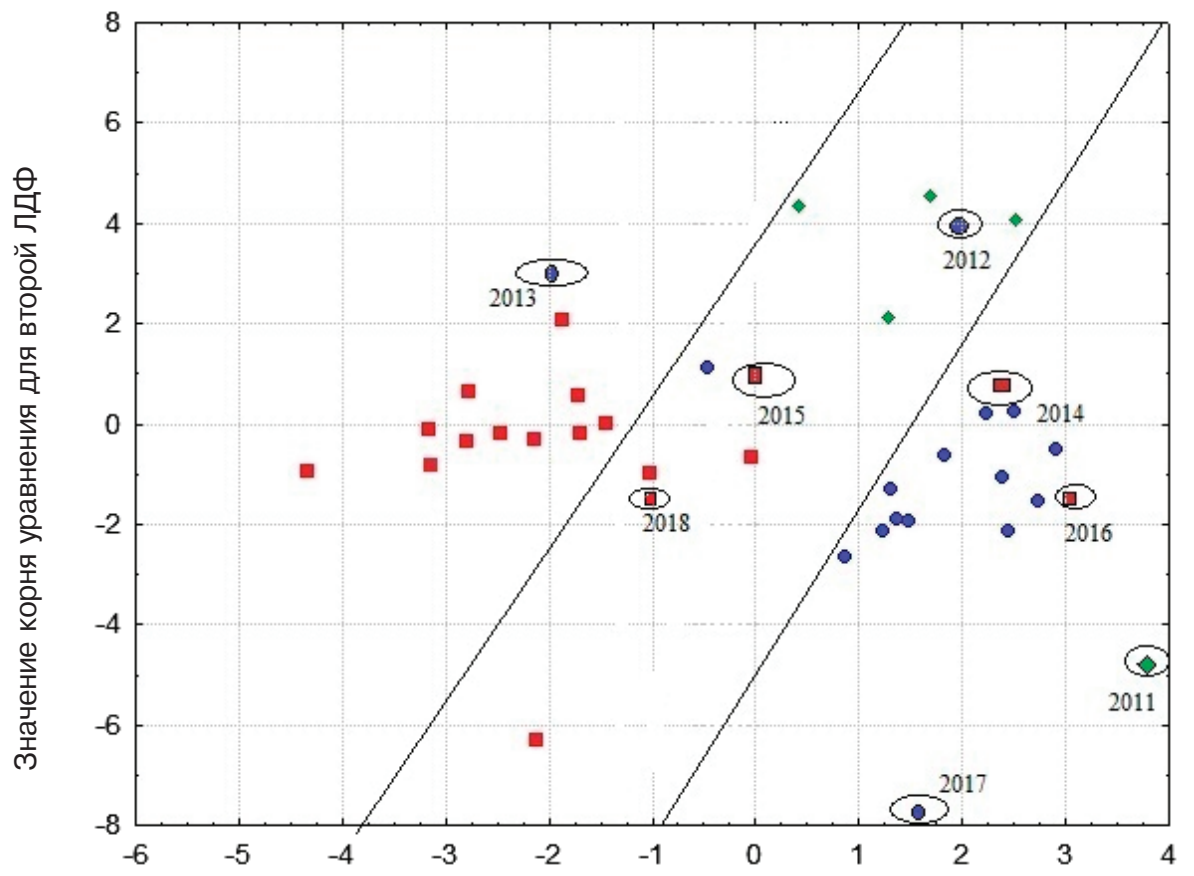

Значение корня уравнения для первой ЛДФ

\section{Pnc. 1.}

Визуализация модели долгосрочного прогноза типа весны по дате перехода среднесуточной температуры воздуха через $0^{\circ} \mathrm{C}$ 
Таблица 3. Матрица сопряженности прогностических и фактических состояний преАиктанта

\begin{tabular}{|l|c|c|c|c|}
\hline \multirow{2}{*}{$\begin{array}{c}\text { Фактически } \\
\text { наблюлалось } \Phi_{i}\end{array}$} & Ранняя $\Pi_{1}$ & Нормальная $\Pi_{2}$ & Поздняя $\Pi_{3}$ & $\sum_{j=1}^{m} n_{1}$ \\
\cline { 2 - 5 } & $12 / 12$ & $-/ 1$ & $-/-$ & $12 / 13$ \\
\hline Ранняя $\Phi_{1}$ & $-/ 2$ & $12 / 13$ & $1 / 1$ & $13 / 16$ \\
\hline Нормальная $\Phi_{2}$ & $2 / 3$ & $1 / 2$ & $4 / 5$ & $7 / 10$ \\
\hline Поздняя $\Phi_{3}$ & $14 / 17$ & $13 / 16$ & $5 / 6$ & $32 / 39$ \\
\hline$\sum_{j=1}^{m} n_{1}$ & & & & \\
\hline
\end{tabular}

С применением матрицы сопряженности можно определить чувствительность модели к редкому явлению $\left(\lambda^{*}=q_{1} \sqrt{ } p_{01}\right.$, где $q_{11}-$ доля оправдавшихся прогнозов редкого явления; $p_{01}-$ доля всех прогнозов редкого явления). Поскольку реже всего наблюдается поздняя дата перехода, определим чувствительность модели с прогнозу именно этой даты. C учетом данных зависимых испытаний $\lambda^{*}$ оказалась равной 0.54 , независимых $-\lambda^{*}=0.50$. Заметим, что наиболее трудно прогнозировать именно редкие явления. «Схватывание» моделью половины редких явлений можно считать весьма удачным.

Рассчитаем ранговую меру связи по Гудмэну и Крускалу $\gamma$, показывающую вероятностную оценку соотношения всех правильных и всех неправильных прогнозов:

$$
\gamma=\frac{s-D}{s+D}
$$

$S$ - общее число пар наблюдений, для которых либо одновременно $i_{1}>i_{2} u j_{1}>j_{2}$, либо, наоборот, $i_{1}<i_{2} u j_{1}<j_{2}$, т.е. когда ранги пар измерений совпадают,

$D$ - общее число пар наблюдений, для которых либо $i_{1}>i_{2} u j_{1}<j_{2}$, либо $i_{1}<i_{2}$ и $j_{1}>j_{2}$, т.е. когда ранги пар измерений не совпадают.

В долях единицы величина этого показателя по зависимым испытаниям составила 0.09 , с учетом независимых - 0.21. Отметим, что с учетом независимых испытаний доля неправильных прогнозов несколько повышается, что вполне обычно для физико-статистических моделей. Однако полученные значения позволяют говорить об очень малой доле неоправдавшихся прогнозов.

В качестве оценки меры мастерства проведено сравнение оправдываемости методического прогноза с оправдываемостью климатического прогноза.

$$
\Delta Q S=Q-Q \mathrm{cl}, Q S=Q / Q \mathrm{cl}
$$

$Q$ - оправдываемость методического прогноза,

$Q \mathrm{cl}$ - оправдываемость климатического прогноза.

Климатический прогноз строился в предположении, что все даты перехода соответствуют климатической норме, т.е. являются нормальными. Оправдываемости и методического, и климатического прогнозов рассчитаны по всему имеющемуся ряду лет. С учетом этих положений $\Delta Q S=36 \%$, а выигрыш методического прогноза по отношению к климатическому составляет 1.85 .

\section{BыBOabl}

1. Применен прием непараметрического дискриминантного анализа для разделения трех групп объектов - раннего, нормального и позднего срока наступления даты перехода через определенный предел. Применение дискриминантной модели для разделения трех групп явлений расширяет возможности физико-статистического направления долгосрочного прогнозирования по более глубокой дифференциации прогнозируемых явлений.

2. Выявлены асинхронные дальние связи между состоянием центров действия атмосферы и сроками наступления даты перехода средней суточной температуры через $0^{\circ} \mathrm{C}$ на юго-востоке Европейской части России. Наиболее информативными для прогноза оказались интенсивность Исландского минимума в январе и географическая локализация его центра в ноябре, а также смещение вдоль круга широты зимнего Азиатского антициклона в ноябре.

3. Получены прогностические разработки по определению будущего характера даты перехода средней суточной температуры через $0^{\circ} \mathrm{C}$ для территории Саратовской области и проведена их визуализация. Оценка прогнозов, составленных по зависимой выборке и с учетом независимых испытаний, показала надежность и хорошие перспективы предлагаемой методики для прогнозирования сезонных гидрометеорологических явлений.

4. Как самостоятельный вывод можно рассматривать указание на необходимость дальнейших методических разработок по оцениванию трехфазных прогнозов.

\section{СПИСОК ПИТЕРАТУРЫ}

1. Адрианова Л.В. 1970. К вопросу прогноза дат наступления сезонных метеорологических явлений в Саратовской области // Вопросы климата и погоды Нижнего Поволжья. Саратов. Вып. 6. С. 54-58. [Adrianova L.V. 1970. K voprosu prognoza dat nastupleniya sezonnykh meteorologicheskikh yavleniy v Saratovskoy oblasti // Voprosy klimata i pogody Nizhnego Povolzh'ya. V. 6. P. 54-58. (In Russian)].

2. Атлас Саратовской области. 1978. М.: Главное управление геодезии и картограсии при совете министров СССР. 14 с. [Atlas Saratovskoy oblasti. 1978. Moscow: Glavnoe upravlenie geodezii i kartografii pri sovete ministrov SSSR. 14 p. (In Russian)]. 
3. Байдал М.Х., Неушкин А.И. 1979. Макроциркудяционный метод и прогноз засух в основных сельскохозяйственных районах страны // Труды ВНИИГМИ-МЦД. Вып. 59. 140 с. [Baydal M. Kh., Neushkin A.l. 1979. Makrotsirkudyatsionnyy metod i prognoz zasukh v osnovnykh sel'skokhozyaystvennykh rayonakh strany // Works of RSRIOHI (Russian Scientific Research Institute of Hydrometeorological Information). V. 59. P. 140. (In Russian)].

4. Вильфанд Р.М., Мартазинова В.Ф., Цепелев В.Ю., Хан В.М., Мироничева Н.П., Елисеев Г.В., Иванова Е.К., Тищенко В.А., Уткузова Д.Н. 2017. Комплексирование синоптико-статистических и гидродинамических прогнозов температуры воздуха на месяц // Метеорология и гидрология. № 8. C. 5 - 17. [Vil'fand R.M., Khan V.M., Eliseev G.V., Tishchenko V.A., Martazinova V.F., Tsepelev V.Y., Mironicheva N.P., Utkuzova D.N., Ivanova E.K. 2017. Integration of synoptic and hydrodynamic monthly air temperature forecasts // Russian Meteorology and Hydrology. V. 42. № 8. P. 485-493.]

5. Вильфанд Р.М., Тищенко В.А., Хан В.М. 2007. Статистический прогноз хода температуры воздуха внутри месяца с использованием выходных данных гидродинамических моделей // Метеорология и гидрология. № 3. С.5-13. [Vil'fand R.M., Tishchenko V.A., Khan V.M. 2007. Statistical forecast of temperature dynamics within month on the basis of hydrodynamic model outputs // Russian Meteorology and Hydrology. V. 32. № 3. P. 147-153.]

6. Данные о срочных значениях температуры воздуха для метеостанций Саратовской области. 2017. ВНИИГМИМЦД. URL: http://meteo.ru/index.html (дата обращения 22.02.2017). [The data of urgent values of air temperature for meteorological stations in Saratovskaya oblast'. 2017. RSRIOHI (Russian Scientific Research Institute of Hydrometeorological Information). URL: http://meteo.ru/ index.html (Access date 22.02.2017). (In Russian)].

7. К Киктев Д.Б., Толстых М.А., Зарипов Р.Б., Круглова Е.Н., Куликова И.А., Тищенко В.А., Хан В.М. 2017. Выпуск детализированных метеорологических прогнозов в рамках деятельности Северо-Евразийского климатического центра (СЕАКЦ) // Труды Гидрометцентра России. Вып. 366. С. 14-28. [Kiktev D.B., Tolstykh M.A., Zaripov R.B., Kruglova E.N., Kulikova I.A., Tishenko V.A., Khan V.M. 2017. Issue of detailed meteorological forecasts in North Eurasian Climate Centre (NEACC) // Proceedings of Hydrometcentre of Russia. V. 366. P. 14-28.]

8. Крыжов В.Н. 2012. Региональная коррекция для северной Евразии глобальных сезонных прогнозов Гидрометцентра России // Метеорология и гидрология. № 5. С. 5-14. [Kryzhov V.N. 2012. Downscaling of the global seasonal forecasts of Hydrometcenter of Russia for north Eurasia // Russian Meteorology and Hydrology. V. 37. № 5. P. 291-297.]

9. Крыжов В. Н. 2003. Связь средних месячной, сезонной и годовой температур воздуха на Севере России с индексами зональной циркуляции зимой // Метеорология и гидрология. № 2. C. 15-28. [Kryzhov V.N. 2003. Connection between monthly mean, seasonal, and annual air temperatures in northern Russia and winter zonal circulation indices // Russian Meteorology and Hydrology. V. 2. P. 9-19.]

10. Мирвис В.М., Мелешко В.П., Львова Т.Ю., Матюгин В.А. 2017. Пятилетний опыт оперативного прогнозирования метеорологических условий на срок до 45 суток на основе модели общей циркуляции атмосферы ГГО (версия MG0-3 T63L25 // Труды Гидрометцентра России. Вып. 366. C. 29-50. [Mirvis V.M., Meleshko V.P., Lvova T.U., Matugin V.A. 2017. Five years experience of operational forecasting meteorological conditions for up to 45 days based on the atmosphere general circulation model of the MGO (version MG0-3 T63L25) // Proceedings of Hydrometcentre of Russia. V. 366. P. 29-50. (In Russian)].

11. Морозова С.В. 2011. Комплексное исследование поведения центров действия атмосферы Атлантико-Евразийного сектора полушария. СПб: Учёные записки РГГМУ. Вып. 21. C. 53-56. [Morozova S.V. 2011. Kompleksnoe issledovanie povedeniya tsentrov deystviya atmosfery Atlantiko-Evraziynogo sektora polushariya. Saint-Petersburg: RSHU (Russian State Hydrometeorological University). V. 21. P. 53-56 (In Russian)].

12. Неушкин А.И., Сидоренков Н.С., Санина А.Т., Иванова Т.Б., Бережная Т.В., Панкратенко Н.В., Макарова М.Е. 2013. Мониторинг общей циркуляции атмосферы. Северное полушарие. Обнинск: ВНИИГМИ-МЦД. 200 с. [Neushkin A.I., Sidorenkov N.S., Sanina A.T., Ivanova Y.B., Bereznaya T.V., Pankratenko N.V., Makarova M.E. 2013 Monitoring obshchey tsirkulyatsii atmosfery. Severnoe polusharie.. Obninsk: RSRIOHI (Russian Scientific Research Institute of Hydrometeorological Information). 200 p. (In Russian)].

13. Руководство по агрометеорологическим прогнозам. Зерновые культуры. 1984. Л.: Гидрометеоиздат. Том I. 309 с. [Rukovodstvo po agrometeorologicheskim prognozam. Zernovye kul'tury. 1984. Leningrad: Hydrometeorological publication. Volume I. 309 p. (In Russian)].

14. Садоков В.П., Козельцева В.Ф., Кузнецова Н.Н. 2012. Определение дат устойчивого перехода средней суточной температуры воздуха через $0,+5{ }^{\circ} \mathrm{C}$, их прогноз и оценка. М.: Тр. Гидрометеорологического НИЦ РФ. Вып. 348. С. 144-152. [Sadokov V.P., Koselzeva V.F., Kusnezova N.N. 2012. Opredelenie dat ustoychivogo perekhoda sredney sutochnoy temperatury vozdukha cherez $0,+5{ }^{\circ} \mathrm{C}$, ikh prognoz i otsenka. Moscow: Trudy Gidrometeorologicheskogo NITs RF V. 348. P. 144-152. (In Russian)].

15. Угрюмов А.И. 2006. Долгосрочные прогнозы погоды. С-Пб: РГГМУ. 82 с. [Ugrumov A.I. 2006. Dolgosrochnye prognozy pogody. Saint-Petersburg: National Russian Hydrometeorological University. 82 p. (In Russian)].

16. Хандожко Л.Т. 2005. Экономическая метеорология. С-Пб: Гидрометеоиздат. 490 с. [Handozko L.T. 2005. Economical meteorology. Saint-Petersburg: Hydrometeorological Publication. 490 p. (In Russian)].

17. Randall D., Curry J. 1998. Status of and outlook for largescale modelling of atmosphere-ice-ocean interactions in the Arctic // BAMS. V. 79. P. 197-219.

18. Wallace J.M., Gutzler D.S. 1981. Teleconnections in the geopotential height field during the Northern hemisphere winter // Mon. Wea. Rev. V.109. P. 784-812.

19. Wilks D.S. 1995. Statistical methods in the atmospheric sciences. Moscow: Academic Press. 467 p. 